\title{
EQUIVARIANT BORDISM AND SMITH THEORY
}

\author{
BY \\ R. E. STONG
}

\begin{abstract}
The relationship between equivariant bordism and Smith homology theory on the category of pairs with involution is studied.
\end{abstract}

1. Introduction. The object of this paper is to analyze the relationship between the homology theories given by equivariant bordism $\mathfrak{N}_{*}^{Z_{2}}(X, A, T)$ and Smith homology theory $H_{*}^{Z_{2}}\left(X, A, T ; Z_{2}\right)$ on the category of pairs with involution. These theories are related in much the same way as ordinary bordism and homology are related. (See for example [1, §8 and $\$ 17]$.)

In $\S 2$, basic definitions will be given, and then it will be shown that assigning to a bordism element $f:(M, \partial M, S) \rightarrow(X, A, T)$ the image of the fundamental Smith theory class of $(M, \partial M, S)$ defines a natural isomorphism

$$
\tilde{\mu}: \mathfrak{N}_{*}^{Z_{2}}(X, A, T) \otimes_{\mathbb{M}_{2} Z_{2}} Z_{2} \stackrel{\cong}{\longrightarrow} H_{*}^{Z_{2}}\left(X, A, T ; Z_{2}\right) .
$$

It should be noted, however, that one cannot have the other isomorphism relation (valid for ordinary bordism)

$$
\mathfrak{N}_{*}^{Z_{2}}(X, A, T) \cong H_{*}^{Z_{2}}\left(X, A, T ; Z_{2}\right) \otimes_{Z_{2}} \mathfrak{N}_{*}^{Z_{2}}
$$

for all $(X, A, T)$ since $Z_{2}$ bordism is not in general a free $\mathfrak{N}_{*}^{Z_{2}}$ module. (If $X=2$ points interchanged by $T$ and $A=\varnothing, \mathfrak{N}_{*}^{Z_{2}}(X, A, T) \cong \mathfrak{N}_{*}$, while $H_{*}^{Z_{2}}(X, A, T)$ $\otimes_{z_{2}} \mathfrak{N}_{*}^{Z_{2}} \cong Z_{2} \otimes_{z_{2}} \mathfrak{N}_{*}^{Z_{2}} \cong \mathfrak{N}_{*}^{Z_{2}}$, and these are not isomorphic as graded groups.)

In $\S 3$, it will be shown that the composite

$$
\mu \circ \tau_{*}: \mathfrak{R}_{*}^{Z_{2}}(X, A, T) \rightarrow H_{*}^{Z_{2}}\left(X \times B O, A \times B O, T \times S ; Z_{2}\right)
$$

is monic, where $\tau_{*}: \mathfrak{R}_{*}^{Z_{2}}(X, A, T) \rightarrow \mathfrak{N}_{*}^{Z_{2}}(X \times B O, A \times B O, T \times S)$ sends $f:(M, \partial M, Q) \rightarrow(X, A, T)$ to $f \times \tau:(M, \partial M, Q) \rightarrow(X \times B O, A \times B O, T \times S)$ with $\tau$ equivariantly classifying the stable tangent bundle of $M$. Thus, the dual Smith cohomology groups

$$
H_{Z_{2}}^{*}\left(X \times B O, A \times B O, T \times S ; Z_{2}\right)
$$

provide characteristic numbers which determine the bordism class. There has been much recent interest in equivariant characteristic numbers (see [5], for example). Much of the effort has been devoted to more difficult cases using modern methods

Received by the editors August 13, 1970.

AMS 1969 subject classifications. Primary 5710; Secondary 5747, 5536.

Key words and phrases. Equivariant bordism, Smith theory.

Copyright (C) 1971, American Mathematical Society 
with equivariant Thom classes and such (in for example the work of T. tom Dieck or G. Hamrick). The approach taken here is much more naive.

I am especially indebted to Professor E. E. Floyd for his interest and encouragement, and for pointing out the existence of the fundamental class in Smith theory. I am also indebted to the Alfred P. Sloan Foundation for financial support during this work.

2. The representation theorem. Let $X$ be a simplicial complex $T: X \rightarrow X$ a simplicial involution $\left(T^{2}=\right.$ identity) and $A \subset X$ an invariant subcomplex $(T A \subset A)$. It will be assumed that $X$ is "finely" triangulated so that the fixed set $F$ of $T$ on $X$ is a subcomplex and the orbit map $\pi: X \rightarrow X / Z_{2}$ is simplicial (E. E. Floyd [3] shows that this may be accomplished by taking the second barycentric subdivision).

Letting $C(X) \otimes Z_{2}$ denote the chains of $X$ with mod 2 coefficients, one has an induced chain map $T_{\#}: C(X) \otimes Z_{2} \rightarrow C(X) \otimes Z_{2}$ with $T_{\#} \circ T_{\#}=1$, and one lets $C^{0}(X)$ denote the subgroup of chains invariant under $T_{\#}\left(T_{\#} \sigma=\sigma\right)$. Since the boundary operator commutes with $T_{\#}$, one has induced a boundary homomorphism ว: $C^{0}(X) \rightarrow C^{0}(X)$, making $\left(C^{0}(X), \partial\right)$ a complex. One lets $C^{0}(X, A)=C^{0}(X) / C^{0}(A)$, and $\partial$ makes this into a chain complex. The Smith homology groups of $(X, A, T)$, $H_{*}^{Z_{2}}\left(X, A, T ; Z_{2}\right)$ are then defined to be the homology groups of the complex $\left(C^{0}(X, A), \partial\right)$.

Now consider a chain $\sigma=\sum a_{i} \Delta^{i}$, where $\Delta^{i}$ are $n$-simplices and $a_{i} \in Z_{2}$. The chain $\sigma$ is invariant under $T_{\#}$ if and only if, for each $n$-simplex $\Delta$, the coefficients of $\Delta$ and $T_{\#} \Delta$ in $\sigma$ are the same. Thus $\sigma$ is a sum of terms $\Delta+T_{\#} \Delta$ (if $T_{\#} \Delta \neq \Delta$ ) and terms $\Delta$ (with $T_{\#} \Delta=\Delta$ ), so that $C^{0}(X)$ decomposes into a direct sum $U \oplus V$, where $U$ is spanned by the $\Delta+T_{\#} \Delta\left(T_{\#} \Delta \neq \Delta\right)$ and $V$ is spanned by the $\Delta$ with $T_{\#} \Delta=\Delta$. Clearly $\partial\left(\Delta+T_{\#} \Delta\right)$ is a sum of terms of the same form for if a face $\Delta^{\prime}$ of $\Delta$ is fixed by $T_{\#}, \Delta^{\prime}$ occurs with coefficient $2 \equiv 0$ in $\partial\left(\Delta+T_{\#} \Delta\right)$, while if $T_{\#} \Delta=\Delta$, then by the "fineness" of the triangulation, each vertex of $\Delta$ must be fixed so each face of $\Delta$ is fixed by $T_{\#}$. Thus $C^{0}(X)$ is the direct sum of the subcomplexes $U$ and $V$, inducing a decomposition of $C^{0}(X, A)$ and $H_{*}^{Z_{2}}\left(X, A, T ; Z_{2}\right)$.

Now let $\rho: C\left(X / Z_{2}\right) \otimes Z_{2} \rightarrow C(X) \otimes Z_{2}$ be defined as follows. If $\Delta^{\prime}$ is a simplex of $X / Z_{2}$, let $\Delta$ be a simplex of $X$ with $\pi \Delta=\Delta^{\prime}$, and let $\rho\left(\Delta^{\prime}\right)=\Delta+T_{\#} \Delta$, which is well defined since $\Delta$ and $T_{\#} \Delta$ are the two possible lifts of $\Delta^{\prime}$. Clearly $\rho$ is a chain map with image $U \subset C^{0}(X)$ and kernel $C(F) \subset C\left(X / Z_{2}\right)$. Thus $\rho$ induces an isomorphism of $C\left(X / Z_{2}\right) / C(F)+C\left(A / Z_{2}\right)=C\left(X / Z_{2}, A / Z_{2} \cup F\right)$ with the $U$ summand of $C^{0}(X, A)$, and the $U$ summand of $H_{*}^{Z_{2}}\left(X, A, T ; Z_{2}\right)$ is identified with $H_{*}\left(X / Z_{2}, A / Z_{2} \cup F ; Z_{2}\right)$. Clearly the inclusion $i: F \rightarrow X$ identifies $C(F)$ with $V$, and so the $V$ summand of $H_{*}^{Z_{2}}\left(X, A, T ; Z_{2}\right)$ is identified with $H_{*}\left(F, F \cap A ; Z_{2}\right)$. This gives the standard result

THEOREM 2.1. $H_{*}^{Z_{2}}\left(X, A, T ; Z_{2}\right) \cong H_{*}\left(X / Z_{2}, A / Z_{2} \cup F ; Z_{2}\right) \oplus H_{*}\left(F, F \cap A ; Z_{2}\right)$.

By using Čech [6], [4] or singular [2] methods to obtain a complex, this may be extended to topological pairs with involution $(X, A, T)$. Similarly using 
Hom $\left(C(X) ; Z_{2}\right)$ one may form Smith cohomology groups dual to the homology groups.

Being given a compact differentiable manifold with boundary $M^{n}$ with differentiable involution $S$, one may triangulate $M$ "finely" so that $S$ is simplicial (by triangulating $M / Z_{2}$ and lifting the triangulation). Clearly the fundamental cycle $\mu=\sum \Delta^{i}$, the sum of all $n$-simplices, is then an invariant chain, defining a fundamental class $[M, \partial M, S] \in H_{n}^{Z_{2}}\left(M, \partial M, S ; Z_{2}\right)$. This lifts $[M, \partial M] \in H_{n}\left(M, \partial M ; Z_{2}\right)$ back along the forgetful homomorphism from Smith theory to ordinary homology.

One then has a natural homorphism

$$
\mu: \mathfrak{R}_{*}^{Z_{2}}(X, A, T) \rightarrow H_{*}^{Z_{2}}\left(X, A, T ; Z_{2}\right)
$$

assigning to the equivariant bordism element $f:(M, \partial M, S) \rightarrow(X, A, T)$ the class $f_{*}[M, \partial M, S]$.

If $b=(B, U) \in \mathfrak{N}_{*}^{Z_{2}}$ and $\alpha=(M, \partial M, S, f) \in \mathfrak{N}_{*}^{Z_{2}}(X, A, T)$, their product $b \alpha=$ $\left(B \times M, B \times \partial M, U \times S, f \circ \pi_{M}\right) \in \mathfrak{N}_{*}^{Z_{2}}(X, A, T)$ is sent by $\mu$ to

$$
f_{*} \circ \pi_{M *}[B \times M, B \times \partial M, U \times S] .
$$

Since $M / Z_{2}$ and its fixed set are complexes of dimension at most that of $M$, Theorem 1 shows that $\pi_{M \cdot}[B \times M, B \times \partial M, U \times S]=0$ if the dimension of $B$ is positive. If the dimension of $B$ is zero, with $B$ consisting of $k$ points, then $\pi_{M}: B \times M \rightarrow M$ is a $k$-fold cover and $\pi_{M}$. sends the fundamental cycle of $B \times M$ to $k$ times that of $M$. Thus $\mu(b \alpha)=k \mu(\alpha)=\varepsilon(b) \cdot \mu(\alpha)$, where $\varepsilon: \mathfrak{R}_{*}^{Z_{2}} \rightarrow Z_{2}$ is the augmentation to $\mathfrak{R}_{*}^{Z_{2}} \cong \mathfrak{N}_{0} \cong Z_{2}$.

Thus $\mu$ induces a natural homomorphism

$$
\tilde{\mu}: \mathfrak{N}_{*}^{Z_{2}}(X, A, T) \otimes_{\mathfrak{R}_{*} Z_{2}} Z_{2} \rightarrow H_{*}^{Z_{2}}\left(X, A, T ; Z_{2}\right) .
$$

Proposition 2.1. $\tilde{\mu}$ is epic.

Proof. It suffices to show that $\tilde{\mu}$ maps onto each of the summands of $H_{*}^{Z_{2}}\left(X, A, T ; Z_{2}\right)$.

If $\alpha \in H_{*}\left(F, F \cap A ; Z_{2}\right)$, there is an ordinary bordism class $f:(N, \partial N) \rightarrow(F, F \cap A)$ with $f_{*}[N, \partial N]=\alpha$. Letting 1 denote the trivial involution on $N, f:(N, \partial N, 1)$ $\rightarrow(X, A, T)$ is an equivariant bordism element with $f_{*}[N, \partial N, 1]=\alpha$.

If $\alpha \in H_{*}\left(X / Z_{2}, A / Z_{2} \cup F ; Z_{2}\right)$, there is an ordinary bordism element $f:(N, \partial N) \rightarrow\left(X / Z_{2}, A / Z_{2} \cup F\right)$ with $f_{*}[N, \partial N]=\alpha$. Using excision and a neighborhood of $F$ which retracts by a deformation to $F$, one may find a double cover $\tilde{N} \stackrel{\pi^{\prime}}{\rightarrow} N$, with covering involution $S$ and an equivariant map $\tilde{f}: \tilde{N} \rightarrow X$ with the diagram

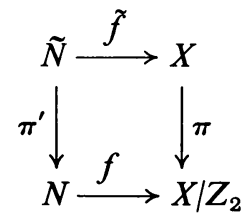

commutative. Then $\tilde{f}(\partial N) \subset A \cup F$ and by applying excision methods to a neighborhood of $A$, one may after a small equivariant deformation if needed find a 
decomposition of $\partial N$ into two invariant submanifolds $\partial N=N_{0} \cup N_{1}$ with $N_{0} \cap N_{1}$ $=\partial N_{0}=\partial N_{1}$ with $\tilde{f}\left(N_{0}\right) \subset A, \tilde{f}\left(N_{1}\right) \subset F$. Let $\hat{N}$ be the quotient of $\tilde{N}$ obtained by identifying $x$ with $S x$ for $x \in N_{1}$, with $\hat{f}: \hat{N} \rightarrow X$ induced by $\tilde{f}, \hat{\pi}: \hat{N} \rightarrow N$ induced by $\pi^{\prime}$ and with involution $\hat{S}$ induced by $S$. Then $\hat{f}:(\hat{N}, \partial \hat{N}, S) \rightarrow(X, A, T)$ is an equivariant bordism element and $\hat{f}_{\#}$ sends the fundamental cycle of $\hat{N}$ to the lift by $\rho$ of $f_{\# \mu}$ where $\mu$ is the fundamental cycle of $N$. Then $\hat{f}_{*}[\hat{N}, \partial \hat{N}, S]$ is $\alpha$ in $H_{*}^{Z_{2}}(X, A, T)$.

Applying an excision to identify $(A \cup F, A)$ with $(F, F \cap A)$, the bordism exact sequence of the triple $(X, A \cup F, A)$ gives an exact sequence

and one has

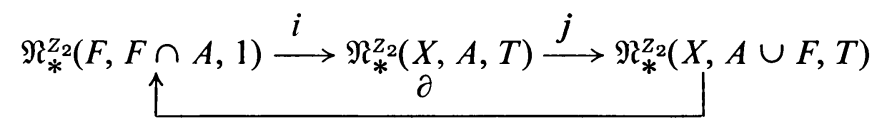

LEMMA 2.1. The homomorphism $\partial$ is zero.

Proof. (1) Applying the fixed point homomorphism

$$
F: \mathfrak{N}_{*}^{Z_{2}}(X, A, T) \rightarrow \oplus_{k=0}^{*} \mathfrak{N}_{*-k}\left(F \times B O_{k},(F \cap A) \times B O_{k}\right)
$$

of [7, §3], $F \circ i$ is a monomorphism onto a direct summand complementary to the $k=1$ term, so $i$ is monic, or $\partial$ is zero.

(2) Any class $\alpha \in \mathfrak{N}_{*}^{Z_{2}}(X, A \cup F, T)$ may be represented by a map $f:(N, \partial N, S)$ $\rightarrow(X, A \cup F, T)$, and by excising a neighborhood of the fixed set of $N$, one may suppose $S$ is free. By excision and a small deformation of $f$ one may suppose $\partial N=N_{0} \cup N_{1}, \partial N_{0}=\partial N_{1}=N_{0} \cap N_{1}$ with $f\left(N_{0}\right) \subset A, f\left(N_{1}\right) \subset F$. Let $\bar{N}$ be the quotient of $N$ by identifying $x$ with $S x$ for $x \in N_{1}$, with $\bar{f}: \bar{N} \rightarrow X$ and $\bar{S}: \bar{N} \rightarrow \bar{N}$ induced by $f$ and $S$. Then $\bar{f}:(\bar{N}, \partial \bar{N}, \bar{S}) \rightarrow(X, A, T)$ has image $\alpha$ under $j$.

Note. This defines a splitting, identifying $\mathfrak{R}_{*}^{Z_{2}}(X, A \cup F, T)$ with the bordism classes for which the fixed point set has codimension 1 .

Special Note. The splitting $\varphi: \mathfrak{N}_{*}^{Z_{2}}(X, A \cup F, T) \rightarrow \mathfrak{N}_{*}^{Z_{2}}(X, A, T)$ just constructed is only a splitting as $\mathfrak{R}_{*}$ modules and not as $\mathfrak{R}_{*}^{Z_{2}}$ modules (see [7, p. 57, note (4)]). However, being given $f:(N, \partial N, S) \rightarrow(X, A \cup F, T)$ representing $\alpha$ with $S$ free and $\partial N=N_{0} \cup N_{1}, f\left(N_{0}\right) \subset A, f\left(N_{1}\right) \subset F$, as above, and $m=[M, U] \in \mathfrak{N}_{*}^{Z_{2}}, \varphi(m \alpha)-m \varphi(\alpha)$ is the class of

$$
g: M \times I \times N_{1} /\{(m, 0, n) \sim(U m, 0, S n),(m, 1, n) \sim(m, 1, S n)\} \rightarrow F
$$

by $g(m, t, n)=f(n)$ and with involution induced by $U \times 1 \times S$. In particular, $f:\left(N_{1}, \partial N_{1}, S\right) \rightarrow(F, F \cap A, 1)$ is a free $Z_{2}$ bordism element and is a sum of products $u \otimes v$ with $u \in \mathfrak{R}_{*}^{Z_{2}}$ (Free)(point) and $v \in \mathfrak{N}_{*}(F, F \cap A)$. Crossing with $M \times I$ and acting as above sends this to a term $u^{\prime} \otimes v$ with $u^{\prime} \in \mathfrak{R}_{*}^{Z_{2}}$, and since $\operatorname{dim} I>0$, $\operatorname{dim} u^{\prime}>0$. Thus $\varphi(m \alpha)-m \varphi(\alpha)$ is decomposable in the $\mathfrak{R}_{*}^{Z_{2}}$ module structure, so that $\varphi$ induces a splitting

for

$$
\varphi^{\prime}: \mathfrak{N}_{*}^{Z_{2}}(X, A \cup F, T) \otimes_{\mathfrak{M} Z_{2}} Z_{2} \rightarrow \mathfrak{N}_{*}^{Z_{2}}(X, A, T) \otimes_{\mathfrak{N}_{*} Z_{2}} Z_{2}
$$

$$
j^{\prime}: \mathfrak{R}_{*}^{Z_{2}}(X, A, T) \otimes_{\mathfrak{N}^{2}} Z_{2} \rightarrow \mathfrak{N}_{*}^{Z_{2}}(X, A \cup F, T) \otimes \mathfrak{N}_{*}^{2} Z_{2} .
$$

Proposition 2.2. $\tilde{\mu}$ is an isomorphism. 
Proof. Tensoring the short exact sequence of the lemma with $Z_{2}$ and applying $\tilde{\mu}$ gives a commutative diagram

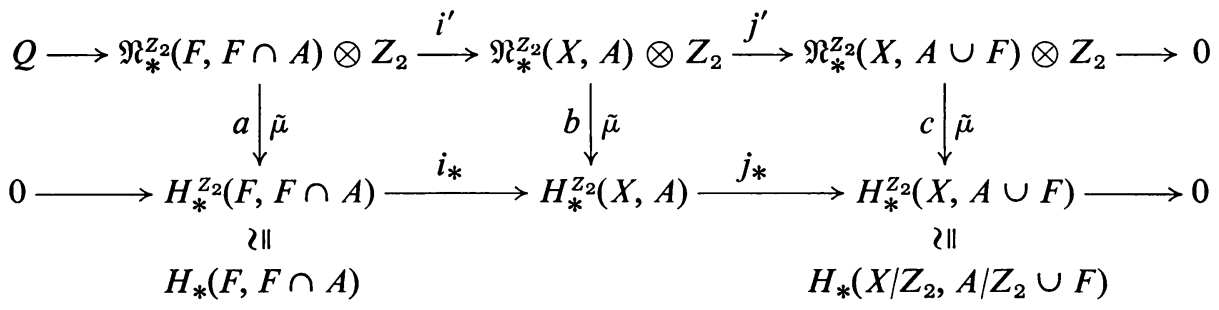

in which the bottom exact sequence is split and gives the direct sum decomposition of Theorem 2.1, with extraneous data being dropped from the notation, and with $Q$ being a Tor-term.

First, $a$ is an isomorphism. Since $\mathfrak{N}_{*}^{Z_{2}}$ is a free $\mathfrak{R}_{*}$ module, the multiplication $\mathfrak{N}_{*}^{Z_{2}} \otimes_{\mathfrak{N}_{*}} \mathfrak{N}_{*}(F, F \cap A) \rightarrow \mathfrak{N}_{*}^{Z_{2}}(F, F \cap A)$ is an isomorphism, being an isomorphism of homology theories on the category of pairs (with trivial action). Thus $\mathfrak{N}_{*}^{Z_{2}}(F, F \cap A) \otimes Z_{2}$ is isomorphic to

$$
Z_{2} \otimes_{\mathfrak{N}_{*}} \mathfrak{R}_{*}^{Z_{2}} \otimes_{\mathfrak{N}_{*}} \mathfrak{R}_{*}(F, F \cap A) \cong Z_{2} \otimes_{\mathfrak{N}_{*}} \mathfrak{R}_{*}(F, F \cap A) \cong H_{*}(F, F \cap A),
$$

and these isomorphisms are just $\tilde{\mu}=a$. In particular, $i_{*} a=b i^{\prime}$ is monic, so $i^{\prime}$ is monic, and the top sequence is short exact.

Next, $c$ is an isomorphism. First, the pair $(X, A \cup F)$ is relatively free as a $Z_{2}$ pair, so $\mathfrak{N}_{*}^{Z_{2}}(X, A \cup F)$ is isomorphic to the free bordism group $\mathfrak{N}_{*}^{Z_{2}}$ (Free) $(X, A \cup F)$. Assigning to a free bordism element $f:(N, \partial N, S) \rightarrow(X, A \cup F, T)$ the map $\bar{f}:\left(N / Z_{2}, \partial N / Z_{2}\right) \rightarrow\left(X / Z_{2}, A / Z_{2} \cup F\right)$ gives an $\mathfrak{N}_{*}$ module isomorphism

$$
\rho: \mathfrak{N}_{*}^{Z_{2}}(X, A \cup F) \rightarrow \mathfrak{N}_{*}\left(X / Z_{2}, A / Z_{2} \cup F\right) .
$$

Clearly $\bar{f}_{*}\left[N / Z_{2}, \partial N / Z_{2}\right]$ in $H_{*}\left(X / Z_{2}, A / Z_{2} \cup F\right)$ represents $\mu(N, \partial N, S, f)$ under the identification with the Smith group, and hence

$$
\hat{\mu}: \mathfrak{R}_{*}^{Z_{2}}(X, A \cup F) \otimes_{\mathfrak{N}_{*}} Z_{2} \rightarrow H_{*}^{Z_{2}}(X, A \cup F)
$$

induced by $\mu$ is an isomorphism. Letting

$$
\theta: \mathfrak{N}_{*}^{Z_{2}}(X, A \cup F) \otimes_{\mathfrak{N}_{*}} Z_{2} \rightarrow \mathfrak{N}_{*}^{Z_{2}}(X, A \cup F) \otimes_{\mathfrak{N}_{*}} Z_{2}
$$

be the quotient, $\theta$ and $\tilde{\mu}$ are epic while $\hat{\mu}=\tilde{\mu} \circ \theta$ is an isomorphism. Thus $\theta$ and $c=\tilde{\mu}$ are isomorphisms.

Note. This indicates that free bordism as $\mathfrak{R}_{*}^{Z_{2}}$ module essentially is given by the $\mathfrak{N}_{*}$ module structure: This is not precise, however.

Finally, since $a$ and $c$ are isomorphisms, so is $b$ by the five lemma, which gives the proposition.

3. The characteristic number theorem. Let $\boldsymbol{R}^{\infty} \oplus \boldsymbol{R}^{\infty}$ denote the countable direct sum of copies of $\boldsymbol{R}$, with involution $s: \boldsymbol{R}^{\infty} \oplus \boldsymbol{R}^{\infty} \rightarrow \boldsymbol{R}^{\infty} \oplus \boldsymbol{R}^{\infty}:(x, y)$ $\rightarrow(x,-y)$, and let $B O_{n}$ be the space of $n$-dimensional subspaces of $\boldsymbol{R}^{\infty} \oplus \boldsymbol{R}^{\infty}$ with 
involution $S$ induced by $s$. Then $\left(B O_{n}, S\right)$ is a classifying space for $n$-plane bundles with involution over reasonably decent spaces with involution. The inclusion $\boldsymbol{R}^{\infty} \oplus \boldsymbol{R}^{\infty} \rightarrow \boldsymbol{R} \oplus \boldsymbol{R}^{\infty} \oplus \boldsymbol{R}^{\infty}:(x, y) \rightarrow(0, x, y)$ and identification of $\boldsymbol{R} \oplus \boldsymbol{R}^{\infty}$ with $\boldsymbol{R}^{\infty}$ (with trivial involutions) gives an equivariant inclusion $i: B O_{n} \rightarrow B O_{n+1}$, so that if $\gamma_{n}$ denotes the universal $n$-plane bundle (with involution) $i^{*}\left(\gamma_{n+1}\right) \cong \gamma_{n} \oplus 1_{+}$, with $1_{+}$being the trivial bundle with trivial involution. Let $(B O, S)$ be the limit of the sequence of $B O_{n}$ 's with these maps.

If $(M, Q)$ is a compact $n$-manifold with boundary, the tangent bundle of $M$, as bundle with involution, is classified by a map $\tau:(M, Q) \rightarrow\left(B O_{n}, S\right)$. If $(M, Q)$ is a regularly imbedded invariant submanifold of $\partial V$ with $\left(V, Q^{\prime}\right)$ an involution, then the tangent bundle of $V$ restricts to $\tau_{M} \oplus 1_{+}$on $M$, which is classified by $i \circ \tau$ : $(M, Q) \rightarrow\left(B O_{n+1}, S\right)$. Thus one has a well-defined homotopy class of maps $\tau_{M}:(M, Q) \rightarrow(B O, S)$ classifying the stable tangent bundle of $M$, and if $(M, Q)$ is regularly imbedded in the boundary of $\left(V, Q^{\prime}\right)$ by $f: M \rightarrow \partial V$, then $\tau_{V} \circ f=\tau_{M}$.

Being given a pair $(X, A, T)$ with involution, one then defines a natural transformation

$$
\tau_{*}: \mathfrak{N}_{*}^{Z_{2}}(X, A, T) \rightarrow \mathfrak{N}_{*}^{Z_{2}}(X \times B O, A \times B O, T \times S)
$$

by sending the class of $f:(M, \partial M, Q) \rightarrow(X, A, T)$ to the class of $f \times \tau_{M}$ : $(M, \partial M, Q) \rightarrow(X \times B O, A \times B O, T \times S)$, where $\tau_{M}$ classifies the stable tangent bundle of $M$ equivariantly.

The remainder of this section will be devoted to a proof of

Proposition 3.1. The composite

$$
\mathfrak{N}_{*}^{Z_{2}}(X, A, T) \stackrel{\tau_{*}}{\longrightarrow} \mathfrak{N}_{*}^{Z_{2}}(X \times B O, A \times B O, T \times S) \stackrel{\mu}{\longrightarrow} H_{*}^{z_{2}}\left(X \times B O, A \times B O, T \times S ; Z_{2}\right)
$$

is monic.

Proof. $\mu \circ \tau_{*}$ is a natural transformation of equivariant homology theories, and so one has a commutative diagram

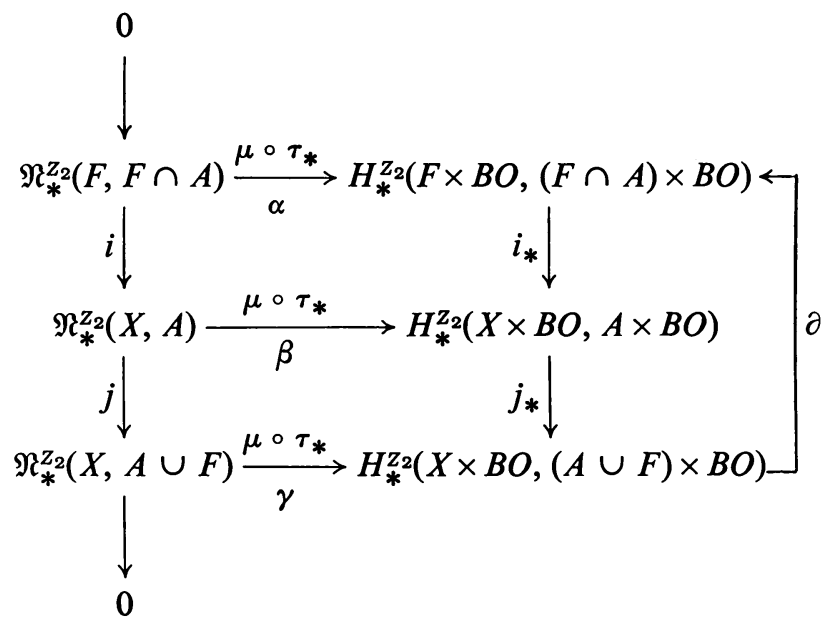


Since $F \times B O$ contains the fixed set of $X \times B O$, the pair $(X \times B O,(A \cup F) \times B O)$ is relatively free, and hence

$$
H_{*}^{Z_{2}}(X \times B O,(A \cup F) \times B O) \cong H_{*}\left((X \times B O) / Z_{2},((A \cup F) \times B O) / Z_{2}\right) .
$$

If $Z$ is a free $Z_{2}$ space, so is $Z \times B O$ and if $\gamma_{r} \rightarrow Z \times B O_{r}$ is the pull back of the universal bundle the quotient $E\left(\gamma_{r}\right) / Z_{2} \rightarrow Z \times B O_{r} / Z_{2}$ is an $r$-plane bundle over $Z \times B O_{r} / Z_{2}$ and is classified by a map $\tilde{\gamma}_{r}: Z \times B O_{r} / Z_{2} \rightarrow B O_{r}$. The bundle $1_{+}$over $Z \times B O_{r}$ gives a trivial bundle over $Z \times B O_{r} / Z_{2}$ and one obtains a map $\tilde{\gamma}: Z \times B O / Z_{2} \rightarrow B O$. Letting $\tilde{\pi}: Z \times B O / Z_{2} \rightarrow Z / Z_{2}$ be induced by projection on $Z$, one has

$$
\tilde{\pi} \times \tilde{\gamma}: Z \times B O / Z_{2} \rightarrow\left(Z / Z_{2}\right) \times B O
$$

and this induces an isomorphism on $Z_{2}$ cohomology. (It is clearly an isomorphism on bordism for the free bordism of $Z \times B O$ may be identified with the bordism of $\left(Z / Z_{2}\right) \times B O$ by sending $f \times \xi: M \rightarrow Z \times B O$ to the class of $\bar{f} \times \bar{\xi}: M / Z_{2} \rightarrow Z / Z_{2} \times B O$ with $\bar{f}$ induced by $f$ and $\xi$ classifying $E(\xi) / Z_{2} \rightarrow M / Z_{2}$.) By excision, this also holds in the relatively free case, so

$$
H_{*}^{Z_{2}}(X \times B O,(A \cup F) \times B O) \cong H_{*}\left(\left(X / Z_{2}\right) \times B O,\left(A / Z_{2} \cup F\right) \times B O\right) .
$$

Now $\mathfrak{N}_{*}^{Z_{2}}(X, A \cup F)$ is isomorphic to the free bordism and so to $\mathfrak{N}_{*}\left(X\left|Z_{2}, A\right| Z_{2} \cup F\right)$, sending a free map $f:(M, \partial M) \rightarrow(X, A \cup F)$ to the class of $\bar{f}:\left(M / Z_{2}, \partial M / Z_{2}\right) \rightarrow\left(X / Z_{2}, A / Z_{2} \cup F\right)$. Applying $\tau_{*}$ and $\tilde{\pi} \times \tilde{\gamma}$ sends $(M, \partial M, f)$ to the image of the fundamental class of

$$
\bar{f} \times \bar{\tau}:\left(M / Z_{2}, \partial M / Z_{2}\right) \rightarrow\left(X / Z_{2} \times B O,\left(A / Z_{2} \cup F\right) \times B O\right)
$$

but $\bar{\tau}$ classifies the stable tangent bundle of $M / Z_{2}$. Thus, the diagram

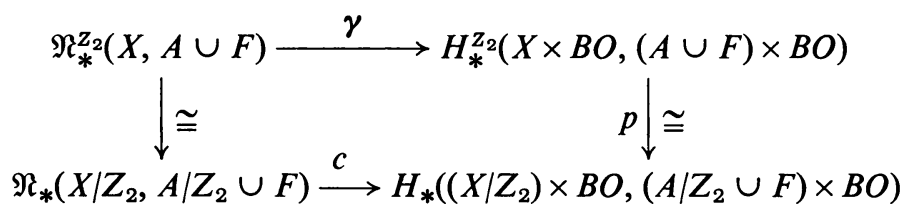

commutes, with $c=\mu \circ \tau_{*}$ in ordinary bordism, which is monic. Thus $\gamma$ is monic.

Next, consider the fixed set of $(B O, S)$. Clearly, $F B O_{n}=\bigcup_{j+k=n} B O_{j} \times B O_{k}$ with $B O_{j} \times B O_{k}$ given by the planes in $\boldsymbol{R}^{\infty} \oplus \boldsymbol{R}^{\infty}$ of the form $U \oplus V, \operatorname{dim} U=j, \operatorname{dim} V=k$. Since $i: B O_{n} \rightarrow B O_{n+1}$ sends $B O_{j} \times B O_{k}$ into $B O_{j+1} \times B O_{k}$, one has $F B O=$ $\bigcup_{k=0}^{\infty} B O \times B O_{k}$. Let $B O^{\prime} \subset B O$ denote the fixed component $B O \times B O_{0} \cong B O$. One then has an equivariant inclusion

$$
q:\left(X \times B O^{\prime}, A \times B O^{\prime}\right) \rightarrow(X \times B O, A \times B O)
$$


with $F\left(X \times B O^{\prime}\right)=F \times B O^{\prime}$. In Smith theory, one then has

$$
\begin{aligned}
& H_{*}^{Z_{2}}\left(X \times B O^{\prime}, A \times B O^{\prime}\right) \stackrel{q_{*}}{\longrightarrow} H_{*}^{Z_{2}}(X \times B O, A \times B O) \\
& \text { 2II } \\
& \downarrow p j_{*} \\
& H_{*}\left(\frac{X}{Z_{2}} \times B O^{\prime},\left(\frac{A}{Z_{2}} \cup F\right) \times B O^{\prime}\right) \quad H_{*}\left(\frac{X}{Z_{2}} \times B O,\left(\frac{A}{Z_{z}} \cup F\right) \times B O\right) \\
& \oplus \\
& H_{*}\left(F \times B O^{\prime},(F \cap A) \times B O^{\prime}\right)
\end{aligned}
$$

and it is immediate that the composite $p j_{*} q_{*}$ on the first summand is just the homomorphism induced by the map

$$
\frac{Z}{Z_{2}} \times B O^{\prime} \stackrel{z}{\longrightarrow} \frac{Z \times B O}{Z_{2}} \stackrel{\tilde{\pi} \times \tilde{\gamma}}{\longrightarrow} \frac{Z}{Z_{2}} \times B O
$$

(on free spaces after excision). Now $B O^{\prime}$ is just a copy of $B O$ and clearly $\tilde{\pi} \circ z$ is the projection on $Z / Z_{2}$ while $\tilde{\gamma} \circ z$ classifies the universal bundle over $B O^{\prime}$, so $(\tilde{\pi} \times \tilde{\gamma}) \circ z$ is an equivalence. Thus $p j_{*} q_{*}$ is epic, but $p$ being an isomorphism, $j_{*}$ is then epic.

The Smith theory sequence of the main diagram is then short exact, $j_{*}$ being epic, with $\gamma$ monic. By standard five lemma type arguments, $\beta$ will be monic if $\alpha$ is monic.

In order to prove $\alpha$ is monic, one must consider $\mathfrak{R}_{*}^{Z_{2}}(F, F \cap A)$. One has an isomorphism

$$
P: \bigoplus_{k=0: k \neq 1}^{*} \mathfrak{R}_{*-k}\left(F \times B O_{k},(F \cap A) \times B O_{k}\right) \rightarrow \mathfrak{R}_{*}^{Z_{2}}(F, F \cap A)
$$

which assigns to $f:(M, \partial M) \rightarrow\left(F \times B O_{k},(F \cap A) \times B O_{k}\right)$ the class obtained as follows. Let $\xi$ be the $k$-plane bundle over $M$ induced by $\pi_{2} \circ f, \bar{M}=$ $D(\xi) /\{x \sim-x \mid x \in S(\xi)\}$ with involution $Q$ induced by -1 in the fibers of $\xi$ and with projection $\pi: \bar{M} \rightarrow M$ induced by projection in $D(\xi)$. Then $P(M, \partial M, f)$ is the class of $\pi_{1} \circ f \circ \pi:(\bar{M}, \partial \bar{M}) \rightarrow(F, F \cap A)$. If $k=0, \bar{M}$ is just $M$ with the trivial involution.

Then $H_{*}^{Z_{2}}(F \times B O,(F \cap A) \times B O)$ may be identified with the direct sum of $R=H_{*}\left(F \times B O / Z_{2},(F \cap A) \times B O / Z_{2} \cup F \times F B O\right)$ and

$$
S=H_{*}(F \times F B O,(F \cap A) \times F B O) .
$$

First, if $\alpha=(M, \partial M, f) \in \mathfrak{R}_{*}\left(F \times B O_{0},(F \cap A) \times B O_{0}\right), \mu \circ \tau_{*} \circ P(\alpha)$ is an element of $S$ since $\bar{M}$ is pointwise fixed by $Q$. Now $S$ decomposes as a direct sum corresponding to the components of $F B O$, with $\tau: \bar{M} \rightarrow F B O$ mapping into the fixed component $B O \times B O_{0}=B O^{\prime}$. Clearly

$\mu \circ \tau_{*} \circ P: \mathfrak{R}_{*}\left(F \times B O_{0},(F \cap A) \times B O_{0}\right) \rightarrow H_{*}\left(F \times B O \times B O_{0},(F \cap A) \times B O \times B O_{0}\right)$ is just $\mu \circ \tau_{*}$ in ordinary bordism, hence is monic. 
Next, if $\alpha=(M, \partial M, f) \in \mathfrak{N}_{*-k}\left(F \times B O_{k},(F \cap A) \times B O_{k}\right), k>1$, the fixed set of $\bar{M}$ is $M$ (image of zero section, codimension $k$ ) and $R P(\xi)$ (image of $S(\xi)$, codimension 1) so that $\mu \circ \tau_{*} \circ P(\alpha)$ lies in the summand $R$. In order to evaluate $\mu \circ \tau_{*} \circ P(\alpha)$ one defines a boundary homomorphism

$$
\partial: R \rightarrow H_{*}\left(F \times F B O \times B Z_{2},(F \cap A) \times F B O \times B Z_{2}\right)=T .
$$

Specifically, one considers $\mathfrak{N}_{*}^{Z_{2}}(F \times B O,(F \cap A) \times B O \cup F \times F B O)$ as the free bordism and takes the boundary to the free bordism

$$
\mathfrak{R}_{*}^{Z_{2}}(\text { Free })(F \times F B O,(F \cap A) \times F B O)
$$

which is isomorphic to $\mathfrak{R}_{*}\left(F \times F B O \times B Z_{2},(F \cap A) \times F B O \times B Z_{2}\right)$. Equivalently $\left(F \times B O \times E Z_{2},((F \cap A) \times B O \cup F \times F B O) \times E Z_{2}\right)$ projects to

$$
(F \times B O,(F \cap A) \times B O \cup F \times F B O)
$$

inducing an isomorphism on Smith theory, and one applies the boundary to

$$
\begin{aligned}
H_{*}^{Z_{2}}(((F \cap A) \times B O \cup F \times F B O) & \left.\times E Z_{2},(F \cap A) \times B O \times E Z_{2}\right) \\
& \cong H_{*}^{Z_{2}}\left(F \times F B O \times E Z_{2},(F \cap A) \times F B O \times E Z_{2}\right) \\
& \cong H_{*}\left(F \times F B O \times B Z_{2},(F \cap A) \times F B O \times B Z_{2}\right)=T .
\end{aligned}
$$

$T$ is, of course, a direct sum over the components of $F B O$.

One now analyzes $\partial \circ \mu \circ \tau_{*} \circ P(\alpha)$. Specifically, $\tau: \bar{M} \rightarrow B O$ is equivariant sending $M$ into the fixed component $B O \times B O_{k}$ to classify $\tau_{M} \oplus \xi$ (the eigenbundles) while $R P(\xi)$ maps into $B O \times B O_{1}$ classifying $\tau_{R P(\xi)} \oplus \lambda$ where $\lambda$ is the canonical line bundle (the normal bundle in $\bar{M}=R P(\xi \oplus 1)$ ). Excising the fixed set in $\bar{M}$ gives a free bordism element $S(\xi) \times[0,1]$ with involution $-1 \times 1$ which maps into $F \times B O$ so that $S(\xi) \times 0 \rightarrow F \times F B O$ by projection on $M$ composed with $\pi_{1} f \times\left(\tau_{M} \times \xi\right)$, hence maps into $F \times B O \times B O_{k}$, and $S(\xi) \times 1 \rightarrow F \times F B O$ is given by projection on $R P(\xi)$ composed with $\left(\pi_{1}(f \circ \pi) \times\left(\tau_{R P(\xi)} \times \lambda\right)\right)$, hence maps into $F \times B O \times B O_{1}$.

Taking the boundary, and excising $S(\xi \mid \partial M) \times[0,1]$ which maps into $(F \cap A) \times B O$, one has the remaining boundary given by $S(\xi) \times\{0,1\}$ and maps as described. Thus $\partial \cdot \mu \cdot \tau_{*} \cdot P(\alpha)$ is the image of the fundamental classes of

$$
(R P(\xi), \partial R P(\xi)) \rightarrow\left(F \times B O \times B O_{k} \times B Z_{2},(F \cap A) \times B O \times B O_{k} \times B Z_{2}\right)
$$

and

$$
(R P(\xi), \partial R P(\xi)) \rightarrow\left(F \times B O \times B O_{1} \times B Z_{2},(F \cap A) \times B O \times B O_{1} \times B Z_{2}\right)
$$

with the maps given by $R P(\xi) \stackrel{\pi}{\rightarrow} M \stackrel{f \times \tau}{\rightarrow} F \times B O, R P(\xi) \stackrel{\pi}{\rightarrow} M \stackrel{\xi}{\rightarrow} B O_{k}, R P(\xi)$ $\stackrel{\lambda}{\rightarrow} B O_{1}$ and $R P(\xi) \rightarrow B Z_{2}$ classifying the double cover by $S(\xi)$.

Letting

$$
\pi_{k}: T \rightarrow H_{*}\left(F \times B O \times B O_{k} \times B Z_{2},(F \cap A) \times B O \times B O_{k} \times B Z_{2}\right)
$$


be the projection for the $k$ th component, one has

$$
\begin{aligned}
\pi_{k} \circ \partial \circ \mu \circ \tau_{*} \circ P: & \mathfrak{N}_{*-k}\left(F \times B O_{k},(F \cap A) \times B O_{k}\right) \\
& \rightarrow H_{*}\left(F \times B O \times B O_{k} \times B Z_{2},(F \cap A) \times B O \times B O_{k} \times B Z_{2}\right)
\end{aligned}
$$

sending $(M, \partial M, f)$ into the image of the fundamental class of $R P(\xi)$ given by the maps

$$
R P(\xi) \stackrel{\pi}{\longrightarrow} M \stackrel{\pi_{1} f \times \tau \times \xi}{\longrightarrow} F \times B O \times B O_{k} \text { and } R P(\xi) \stackrel{\sigma}{\longrightarrow} B Z_{2}
$$

classifying the double covers $S(\xi)$. To see that $\pi_{k} \circ \partial \circ \mu \circ \tau_{*} \circ P$ is monic, let $c \in H^{1}\left(B Z_{2}, Z_{2}\right)$ denote the generator, so that $H^{*}\left(R P(\xi), \partial R P(\xi) ; Z_{2}\right)$ is the free $H^{*}\left(M, \partial M ; Z_{2}\right)$ module via $\pi^{*}$ on $1, \sigma^{*}(c), \ldots, \sigma^{*}(c)^{k-1}$. Then for any $x \in$ $H^{*}\left((F, F \cap A) \times B O \times B O_{k}\right)$,

$$
\begin{aligned}
\left\langle x \otimes c^{k-1}, \pi_{k} \partial \mu \tau_{*} P(\alpha)\right\rangle & =\left\langle\pi^{*}\left(\pi_{1} f \times \tau \times \xi\right)^{*}(x) \cup \sigma^{*}(c)^{k-1},[R P(\xi), \partial R P(\xi)]\right\rangle \\
& =\left\langle\left(\pi_{1} f \times \tau \times \xi\right)^{*}(x),[M, \partial M]\right\rangle
\end{aligned}
$$

but the homomorphism

$$
\mathfrak{R}_{*-k}\left(F \times B O_{k},(F \cap A) \times B O_{k}\right) \rightarrow H_{*}\left(F \times B O \times B O_{k},(F \cap A) \times B O \times B O_{k}\right)
$$

sending $(M, \partial M, f)$ to $\left(\pi_{1} f \times \tau \times \xi\right)_{*}[M, \partial M]$ is just $\mu \circ \tau_{*}$ in ordinary bordism, which is monic. Since homology and cohomology are dual, the numbers $\left\langle x \otimes c^{k-1}, \pi_{k} \partial \mu \tau_{*} P(\alpha)\right\rangle$ then determine $\alpha$.

Thus, the direct sum of the homomorphisms $\pi_{k} \circ \partial \circ \mu \circ \tau_{*} \circ P$ is monic, so $\partial \circ \mu \circ \tau_{*} \circ P$ is monic to $R$ on the $k \neq 0$ summand. Thus $\mu \circ \tau_{*} \circ P$ is monic to $R \oplus S$, and since $P$ is epic, $\mu \circ \tau_{*}=\alpha$ is monic.

This completes the proof of the proposition.

\section{REFERENCES}

1. P. E. Conner and E. E. Floyd, Differentiable periodic maps, Ergebnisse der Mathematik und ihrer Grenzgebiete, Band 33, Academic Press, New York; Springer-Verlag, Berlin, 1964. MR 31 \#750.

2. S. Eilenberg, Homology of spaces with operators. I, Trans. Amer. Math. Soc. 61 (1947), 378-417; errata, ibid. 62 (1947), 548. MR 9, 52.

3. E. E. Floyd, Orbit spaces of finite transformation groups. I, Duke Math. J. 20 (1953), 563-567. MR 15, 456.

4. - "Periodic maps via Smith theory," in A. Borel, Seminar on transformation groups, Ann. of Math. Studies, no. 46, Princeton Univ. Press, Princeton, N. J., 1960. MR 22 \#7129.

5. C. N. Lee and A. G. Wasserman, Equivariant characteristic numbers, Notices Amer. Math. Soc. 17 (1970), 254. Abstract \#672-592.

6. P. A. Smith, "Fixed points of periodic transformations," in S. Lefschetz, Algebraic topology, Amer. Math. Soc. Colloq. Publ., vol. 27, Amer. Math. Soc., Providence, R. I., 1942. MR 4, 84.

7. R. E. Stong, Bordism and involutions, Ann. of Math. (2) 90 (1969), 47-74. MR 39 \#3503.

UNIVERSITY OF VIRGINIA,

Charlottesville, Virginia 22904 